\title{
Instrumento WHOQOL-100 e políticas públicas: avaliação da qualidade de vida de população alvo de política habitacional
}

\section{WHOQOL-IOO and public policies: assessing the quality of life of people assisted by housing policies}

\author{
Beatriz Ferreira de Carvalho ${ }^{a}$ \\ (i) https://orcid.org/0000-0002-6390-2445 \\ E-mail: beatrizcarvalhoı82ळhotmail.com \\ Carla Caroline Inocêncio ${ }^{a}$ \\ (iD) https://orcid.org/0000-0003-1801-4745 \\ E-mail: carlacarolinecciळgmail.com

\section{Eduardo Guadagnin ${ }^{b}$} \\ (D) https://orcid.org/0000-0002-1985-1417 \\ E-mail: eduguadsjcœgmail.com

\section{Edvaldo Amorim ${ }^{a}$} \\ (D) https://orcid.org/0000-0001-8084-3814 \\ E-mail: amorimœunivap.br

\section{Paula Vilhena Carnevale Vianna ${ }^{c}$} \\ (D) https://orcid.org/0000-0002-2266-3812 \\ E-mail: paulacarnevaleœuol.com.br \\ a Universidade do Vale do Paraíba (UNIVAP). Faculdade de \\ Ciências da Saúde (FCS). Instituto de Pesquisa e Desenvolvimento. \\ São José dos Campos, SP, Brasil. \\ ${ }^{b}$ Universidade do Vale do Paraíba (UNIVAP). Faculdade de \\ Educação e Artes (FEA). Instituto de Pesquisa e Desenvolvimento. \\ São José dos Campos, SP, Brasil. \\ ' Universidade do Vale do Paraíba (UNIVAP). Programa de \\ Planejamento Urbano e Regional (PLUR). Universidade Anhembi \\ Morumbi. São José dos Campos, SP, Brasil
}

\section{Resumo}

O ambiente é um dos determinantes sociais da saúde. No Brasil, as habitações sociais, financiadas por políticas públicas, tendem a ser erguidas em espaços desprovidos de infraestrutura urbana e a posse da casa se acompanha de incertezas na condução da vida cotidiana. Este trabalho visa investigar o uso do questionário de qualidade de vida WHOQOL - 100 como instrumento de análise da qualidade de vida de uma população atendida por política habitacional, integrando os campos da saúde coletiva e do planejamento urbano. 0 questionário foi respondido por 101 moradores de um conjunto habitacional de São José dos Campos, um município paulista de grande porte. Encontraram-se baixos escores de autoavaliação de qualidade de vida, ambiente e subjetividade comparados aos dados disponíveis em publicações acadêmicas que avaliam, no geral, populações adoecidas. Os resultados permitiram avaliar de modo objetivo a relação entre ambiente e qualidade de vida, subsidiando a formulação e implantação de políticas sociais integradas às habitacionais para garantia dos direitos sociais de populações carentes. Palavras-chave: Qualidade de Vida; Política Pública; Habitação Social; Subjetividade; Vulnerabilidade.

\section{Correspondência}

Paula Vilhena Carnevale Vianna

Av. Deputado Benedito Matarazzo, 6.070. São Jose dos Campos, SP, Brasil. CEP 12230-002. 


\section{Introdução}

The environment is one of the social determinants of health. In Brazil, social housing, financed by housing policies, tends to be built in spaces devoid of urban infrastructure and home ownership is accompanied by uncertainties in the conduct of daily life. This work aims to investigate the use of the WHOQOL - 10o quality of life questionnaire as an instrument for analyzing the quality of life of people attended by housing policy, integrating the fields of public health and urban planning, especially with regard to housing policies. The questionnaire was applied to 101 residents of a housing complex in São José dos Campos, a large city in São Paulo. Low self-assessment scores for quality of life, environment and subjectivity were found, compared to data available in academic publications, which assess, in general, sick populations. The results allowed, in the studied housing complex, to objectively assess the relationship between environment and quality of life, subsidizing the formulation and implementation of social policies integrated with housing policies to guarantee the social rights of the target populations of housing policies.

Keywords: Quality of Life; Public Policy; Social Housing; Mental Health; Vulnerability.
Este estudo foi motivado por questões levantadas no trabalho de campo de uma disciplina de extensão universitária curricular para os anos iniciais de cursos da área da saúde, em uma universidade comunitária. Docentes e discentes, avaliando a saúde da população que habitava um conjunto habitacional entregue pelo poder público havia sete anos, identificaram o ambiente como fator determinante da saúde, individual e coletiva dos habitantes do residencial. O comprometimento da subjetividade dos indivíduos, a fragilidade dos laços sociais e a dificuldade de acesso às políticas públicas pareciam comprometer a saúde e qualidade de vida dos habitantes, sendo também indicativos de que as políticas sociais dirigidas a essa população não teriam alcançado seus fins. A equipe de Saúde da Família do território combinava a percepção da precariedade da condição de vida com preconcepções e juízos morais acerca dos hábitos, costumes e escolhas dos moradores, por vezes negativamente reforçados em ações conjuntas com a assistência social.

Políticas sociais têm como finalidade promover a seguridade pública e o bem-estar dos cidadãos. Saúde e habitação, direitos sociais constitucionais, possuem uma relação intrínseca, sendo a habitação, comprovadamente, um fator determinante para a saúde dos indivíduos e populações (WHO, 2010). Nessa ótica, a efetividade das políticas habitacionais pode ser avaliada pela constatação da melhoria da qualidade de vida proporcionada às pessoas por elas atendidas.

No Brasil, os referenciais emancipatórios que fundamentam as políticas sociais, inspiradas no modelo dos Estados de Bem-Estar Social, nem sempre são observados na implantação das políticas, muitas vezes reduzidas ao provimento dos bens e/ou serviços. A incipiente prática de avaliação institucional é elemento adicional a dificultar a análise da implantação de políticas sociais e sua influência sobre o bem-estar dos cidadãos.

As políticas públicas habitacionais têm trajetória irregular. Instituídas no país na década de 1960, por governos militares, financiadas pelo Sistema Financeiro da Habitação e pelo Banco Nacional da Habitação, foram marcadas pelo baixo financiamento 
e valorização do investimento privado, pela dificuldade da aquisição de terras urbanizadas a preços acessíveis e pelo caráter clientelista das políticas (Ferreira, 2012). O movimento pela moradia, na década de 1980, reinseriu o tema na Constituição, estabelecendo o conceito de função social da propriedade e a responsabilidade dos munícipios pela política territorial, reafirmada no Estatuto da Cidade, de 2001. O programa Minha Casa Minha Vida, lançado em 2009, embora tenha representado significativo avanço no setor por prever recursos e ter como alvo a população de renda muito baixa, em parceria com investimentos do setor privado, enfrenta obstáculos relacionados à trajetória da política, como o acesso à terra bem localizada, com infraestrutura urbana e disponibilidade de serviços essenciais (Ferreira, 2012).

Pela natureza federalista do Brasil, os programas habitacionais das 27 unidades federativas que compõem o país diferem. No estado de São Paulo, o órgão público responsável pela política habitacional é a Companhia de Desenvolvimento Habitacional e Urbano (CDHU), instituída em meados da década de 1990 (CDHU, [2011]). A CDHU executa os programas habitacionais no estado, em parceria com os municípios, para o atendimento da população de baixa renda, concentrada, prioritariamente, na faixa de um a três salários-mínimos, público que representa uma das demandas mais prementes da sociedade. O plano estadual de habitação de 2011-2023 calculou em 1,26 milhão o déficit de moradias, acrescido de 3,19 milhões de moradias inadequadas, definidas como

moradias que apresentam carências diversas que podem ser solucionadas [...] por meio de reforma, ampliação ou adequação de unidades habitacionais, associadas às ações de urbanização, recuperação urbana e regularização fundiária, conforme o caso. (A Secretaria..., 2020)

Unidade de produção humana, o conceito de habitação, portanto, não se limita à estrutura física da casa, mas constitui parte de um todo que inclui o entorno e as pessoas contextualizadas. O Relatório sobre Habitação da Organização Mundial de Saúde (OMS) (WHO, 2010) apresenta os quatro aspectos que compõem o conceito: a casa (house), correspondente à estrutura física; o lar (home), à estrutura econômica, social e cultural estabelecida pela família residente; o bairro (neighbourhood), abrangendo ruas, lojas, igrejas, escolas, área verde e de recreação, transporte etc., que circundam a casa; e, por fim, a comunidade (community), incluindo os que moram, trabalham ou prestam serviços no bairro.

Na moradia e no seu entorno, com maior ou menor acesso a bens e serviços, as pessoas analisam suas vidas e as percebem como tendo maior ou menor qualidade. A OMS define qualidade de vida como “a percepção do indivíduo de sua posição na vida no contexto da cultura e sistema de valores em que vive e em relação aos seus objetivos, expectativas, padrões e preocupações" (WHO, 1997, p. 1, tradução nossa).

A habitação, considerada nessa acepção ampliada, é fator influenciador da manutenção da qualidade de vida do indivíduo. É, igualmente, elemento relevante para a caracterização da vulnerabilidade, definida por Kaztman (200o, p. 294, tradução nossa) como o "conjunto de recursos, materiais e imateriais, sobre os quais os indivíduos e os lugares têm controle, e cuja mobilização permite melhorar sua situação de bem estar, evitar a deterioração de suas condições de vida ou, ainda, diminuir sua vulnerabilidade”. O Relatório do Desenvolvimento Humano do Programa das Nações Unidas para o Desenvolvimento (PNUD, 2014) classifica como vulneráveis, seja pelas condições socioeconômicas ou por transformações ambientais, pessoas em situação de pobreza e privação extremas. Mulheres, crianças, idosos e pessoas com deficiência compõem esse grupo social. O relatório enfatiza a importância do investimento em medidas e políticas que aumentem a resiliência e as capacidades daqueles que, de outra forma, permanecerão indefinidamente vulneráveis.

Há várias definições para o termo resiliência no campo das ciências sociais. Segundo Grotberg (2013, p. 20), resiliência é a “capacidade humana para enfrentar, sobrepor-se e ser fortalecido ou transformado por experiências de adversidade”. Ungar, Brown e Liebenberg (2008, p. 3) apontam a necessidade de entender sua associação com a "capacidade do indivíduo em navegar [...] seu caminho em direção a recursos de bem-estar assim como a capacidade de suas comunidades oferecerem esses recursos de formas culturalmente significativas". 
Populações vulneráveis, por sua condição socioeconômica, situação ambiental ou, frequentemente, por uma sobreposição de ambas as condições, são a população alvo de políticas habitacionais. A realocação se caracteriza por uma ruptura no curso de vida dos indivíduos e sua rede de relações, incluindo os familiares. Essa ruptura influencia a percepção de bem-estar e qualidade de vida numa perspectiva multidimensional, constituída por parâmetros objetivos e subjetivos, que podem ser mensurados. Nos Estados Unidos, a avaliação sistemática do impacto da habitação sobre a saúde (impact health assessment) é um campo interdisciplinar crescente de estudos e pesquisa aplicada, que, ao revelar a relação entre saúde e habitação com base em evidência científica, fornece subsídios técnicos aos tomadores de decisão e favorece escolhas saudáveis nas políticas, programas e projetos habitacionais e de desenvolvimento urbano. A compreensão e integração dos conceitos de saúde pública nas decisões habitacionais melhora a saúde dos habitantes e fortalece a gestão dos recursos públicos (NCHH, 2016).

Uma das formas de avaliar a qualidade de vida, nessa perspectiva, é o questionário WHOQOL-10o, desenvolvido pelo Grupo de Qualidade de Vida da OMS. $O$ instrumento respeita as diferenças culturais e avalia a qualidade de vida dos sujeitos a partir de seis domínios (físico, psicológico, nível de independência, relações sociais, meio ambiente e espiritualidade/ religiosidade e crenças pessoais [WHO, 1997]). O questionário foi validado para o português ${ }^{1} \mathrm{e}$ vem sendo aplicado no Brasil para a avaliação de qualidade de vida, especialmente em portadores de doenças crônicas. O uso do questionário na formulação e monitoramento de políticas públicas, previsto no manual orientador (WHO, 1997), tem sido pouco explorado.

Este artigo investiga a percepção de qualidade de vida de uma população atendida por uma política pública habitacional, residente em um conjunto habitacional em São José dos Campos, um município paulista de grande porte. O objetivo do trabalho é avaliar, para o caso em questão, o potencial do questionário WHOQOL-10o como um instrumento avaliador de políticas públicas, considerando a articulação entre os campos da saúde e do planejamento urbano, mais especificamente, da política habitacional.

\section{Métodos}

A pesquisa é de natureza exploratória, quantitativa, e tem como procedimento técnico a aplicação de dois questionários a 101 domicílios do conjunto habitacional Residencial Caminhos da Montanha, localizado em um município de grande porte paulista. O questionário WHOQOL, elaborado pela OMS e traduzido e validado para o português, ${ }^{2}$ foi utilizado para avaliar a qualidade de vida dos moradores. Um questionário padronizado pela Defensoria Pública do município permitiu caracterizar os aspectos socioeconômicos da população.

Os dados foram obtidos por entrevistas entre os meses de agosto e setembro de 2017. O WHOQOL-100 é um instrumento autoexplicativo e de autoavaliação. A aplicação seguiu as orientações de Fleck et al. (1999): (1) aplicação assistida - para evitar a alteração do sentido original da questão, o entrevistador relia a pergunta de forma lenta quando o respondente não entendia o significado de alguma pergunta ou palavra, sem utilizar sinônimos ou explicar de outra forma a questão; e (2) aplicação administrada o questionário foi lido pelo entrevistador quando o respondente não tinha condições de ler.

Foram incluídos moradores residentes do conjunto habitacional de estudo com mais de 18 anos de idade. A amostra foi estratificada por local de moradia, alternando-se adultos e jovens de ambos os sexos. Todos os participantes assinaram o Termo de Consentimento Livre e Esclarecido. O estudo foi aprovado pelo Comitê de Ética em Pesquisa da Universidade do Vale do Paraíba em 27/04/2017, parecer 2.449 .546 .

1 QUALIDEP. Versão em português dos instrumentos de avaliação da qualidade de vida (WHOQOL-10o). 1998. Disponível em: <https://bit.ly/3msjj4W >. Acesso em: 11 jun. 2016.

2 QUALIDEP. Versão em português dos instrumentos de avaliação da qualidade de vida (WHOQOL-10o). 1998. Disponível em: <https://bit.ly/3msjj4W >. Acesso em: 11 jun. 2016. 
Em 2010, o município possuía 629.921 habitantes e Índice de Desenvolvimento Humano (IDHM) o,807 (muito alto). A cidade é polo de região metropolitana e possui política habitacional implementada desde o final da década de 1970.

A população avaliada nessa pesquisa habita um conjunto residencial situado na área norte da cidade, em transição para a zona rural. O empreendimento, inaugurado em 2011, foi projetado e erguido em parceria entre a CDHU e o município, tem uma única via de acesso e ocupa uma área caracterizada como urbano-rural, isolada do entorno do bairro. São 256 domicílios, habitados por aproximadamente 800 pessoas (Figura 1). Uma pendência da prefeitura junto à Companhia Ambiental do Estado de São Paulo no cartório de registro de imóveis impede a regularização da posse, e os moradores ainda não possuem escritura do imóvel. A falta de documentação e a localização do conjunto no entorno de uma área de proteção ambiental geram constantes boatos de incerteza da posse definitiva.

\section{Figura I - Localização do Residencial Caminhos da Montanha, SJC, SP, Brasil.}

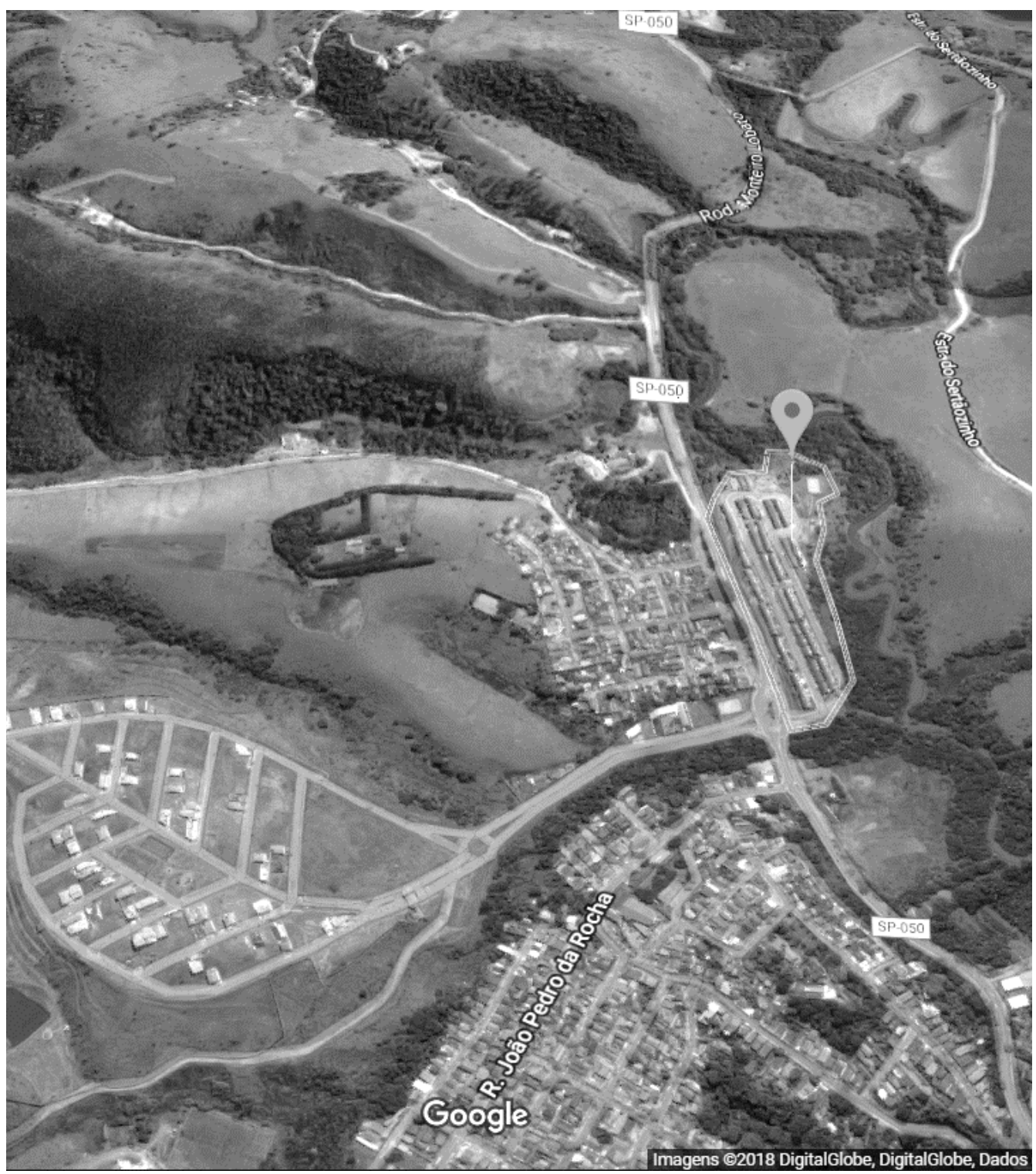




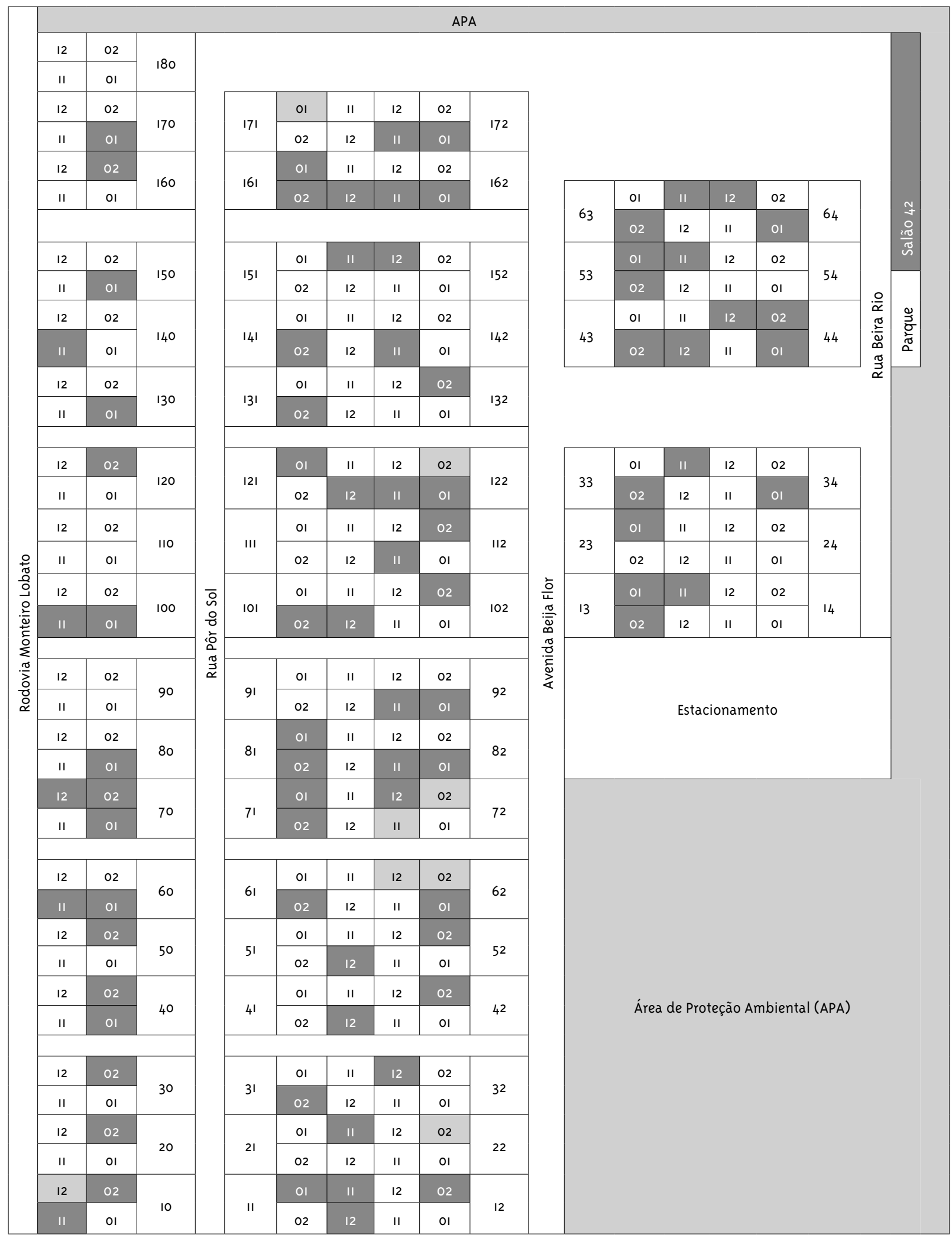

Fonte: Google Maps

Legenda: cinza escuro corresponde a um questionário aplicado na residência e cinza claro a dois questionários. 
O instrumento WHOQOL-10o é qualitativo ordinal de autorresposta. Contém 100 afirmações de respostas em escala do tipo Likert, graduada em cinco níveis: intensidade (nada/extremamente); capacidade (nada/completamente); frequência (nunca/sempre) ou avaliação (muito insatisfeito/muito satisfeito; muito ruim/muito bom). A pessoa indica o grau de concordância com a questão em 1 a 5 , sendo 1 a menor concordância e 5 a maior. Cada faceta corresponde ao somatório dos escores de quatro questões que constitui, por sua vez, os domínios, correspondentes à média entre suas facetas.

O questionário avalia a saúde e qualidade de vida geral e é multidimensional, abrangendo seis domínios: físico, psicológico, nível de independência, relações sociais, meio ambiente e espiritualidade, religiosidade e crenças pessoais (ERCP). Os domínios são divididos em 24 facetas (Tabela 1). Cada faceta é composta por quatro afirmações. Uma $25^{\text {a }}$ faceta, "Qualidade de vida global e percepção geral da saúde”, não inserida em domínio específico, avalia a saúde e qualidade de vida do ponto de vista do respondente. 0 estudo privilegiará a análise dos domínios Ambiente (pela relação direta com a política habitacional) e Psicológico, que motivou a pesquisa.

\section{Tabela I - Domínios e facetas do WHOQOL- 100}

Domínio I-Domínio físico
1. Dor e desconforto
2. Energia e fadiga
3. Sono e repouso
Domínio II - Domínio psicológico
4. Sentimentos positivos
5. Pensar, aprender, memória e concentração
6. Autoestima
7. Imagem corporal e aparência
8. Sentimentos negativos
Domínio III - Nível de independência
10. Atividades da vida cotidiana

\section{Tabela I-Continuação}

II. Dependência de medicação ou de tratamentos

12. Capacidade de trabalho

Domínio IV - Relações sociais

13. Relações pessoais

14. Suporte (apoio) social

15. Atividade sexual

Domínio V-Ambiente

16. Segurança física e proteção

17. Ambiente no lar

18. Recursos financeiros

19. Cuidados de saúde e sociais: disponibilidade e qualidade

20. Oportunidades de adquirir novas informações e habilidades

21. Participação em, e oportunidades de recreação/lazer

22. Ambiente físico: (poluição/ruído/trânsito/clima)

23. Transporte

Domínio $\mathrm{VI}$ - Aspectos espirituais/religião/crenças

pessoais (ERCP)

24. Espiritualidade/religião/crenças pessoais

O questionário socioeconômico foi construído pelos pesquisadores a partir de questionário elaborado pela Defensoria Pública da cidade para complementar as informações obtidas pelo WHOQOL-10o. Esse questionário coletou informações sociodemográficas e socioeconômicas.

A análise dos dados foi realizada a partir do cálculo dos escores do questionário WHOQOL-10o, conforme orientado no manual da OMS (WHO, 1997). Utilizou-se o software Excel para combinar as duas planilhas para organização e análise descritiva de dados. Os domínios e facetas foram agrupados, os valores das facetas e questões de caráter negativo foram invertidos para padronizar todas as respostas do instrumento, de que forma que a resposta mais negativa seja um e a mais positiva seja cinco. Utilizou-se a fórmula de sintaxe padrão indicada no documento de orientação de análise 
para converter a pontuação em escala de zero a 100, sendo zero correspondente ao pior estado geral de saúde e 100 ao melhor estado de saúde. ${ }^{3}$ A estatística analítica foi operada no software Minitab, no qual foram realizados o teste ANOVA para a correlação entre as médias do WHOQOL-10o e as variáveis socioeconômicas e o teste de Pearson para a correlação entre os domínios. Estabeleceu-se o nível de confiança em $95 \%$.

\section{Resultados}

\section{População de estudo}

A amostra totalizou 101 respondentes, cuja idade variou de 18 a 83 anos (média, 41 anos) com predomínio de mulheres (71,3\%) e de paulistas (73,6\%) e, entre estes, destacaram-se os nascidos no próprio munícipio (83,58\%). Em relação ao nível de instrução, 32,7\% da população apresentava menos de nove anos de estudo, 39,6\% de nove a 12 anos de estudo e $27,7 \%$ mais de 12 anos de estudo.

Entre aqueles com ensino fundamental incompleto, 63,0\% possuíam mais de 40 anos. Observa-se, na população jovem (18 a 25 anos), discreta predominância do nível de instrução fundamental completo e ausência de analfabetismo. Quanto à renda, 87,3\% recebiam de zero a dois salários-mínimos. Pela caracterização econômica segundo o critério de Classificação Econômica Brasil, 13,8\% dos respondentes pertencem à classe $\mathrm{D}$ e $87,2 \%$ à classe $\mathrm{E}$.
Em relação à empregabilidade, $17,8 \%$ trabalhavam com registro em carteira e $40 \%$ eram desempregados, sendo o índice mais alto no sexo feminino $(43,9 \%)$ em comparação ao sexo masculino $(29,2 \%)$, e alto percentual em todas as faixas etárias.

Os vínculos informais distribuem-se por uma gama de ocupações de baixo retorno financeiro e pouco valorizadas socialmente, sendo, para as mulheres, o trabalho do lar a ocupação mais comum (48,1\%). As ocupações são identificadas na área da saúde, do comércio informal e de serviços.

A maioria dos participantes veio da região norte do município, a mesma onde o residencial se localiza (72,1\%). A maior parte das realocações foi por situações de risco ambiental (71,9\%), seguida de programas de infraestrutura urbana $(15,6 \%)$ e baixa renda (12,5\%). Os apartamentos de $50 \mathrm{~m}^{2}$ contendo sala, dois quartos, banheiro, cozinha e área de serviço, abrigam de uma (5,9\% dos domicílios) a mais de cinco pessoas (16,8\%), sendo a maior faixa a de dois a quatro moradores por domicílio (57,4\%).

\section{WHOQOL-100 para avaliação da qualidade de vida}

A qualidade de vida geral recebeu o escore de 59,84. Considerando-se os seis domínios do questionário, o Domínio ERCP apresentou escore mais de 20 pontos acima do escore geral de qualidade de vida e o Domínio Ambiente foi o domínio mais mal avaliado, seguido do Físico, sendo ambos inferiores ao escore geral de qualidade de vida (Figura 2).

Figura 2 - Médias das respostas do questionário WHOQOL-100, por domínio, Conjunto Habitacional Caminho das Montanhas, São José dos Campos/SP, ago.-set. $2017 \quad(n=101)$

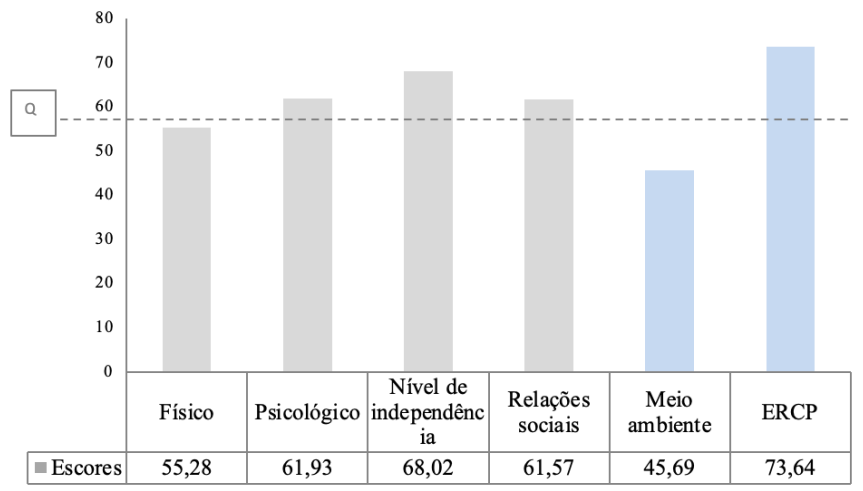

3 QUALIDEP. Versão em português dos instrumentos de avaliação da qualidade de vida (WHOQOL-10o). 1998. Disponível em: <https://bit.ly/3msjj4W >. Acesso em: 11 jun. 2016. 
A análise pelo coeficiente de Pearson mostrou forte correlação entre o Domínio Ambiente e o Domínio Psicológico (o,665221) e moderada entre os Domínios Relações Sociais e Físico (o,438034), o que reforça a adequação da escolha dos domínios selecionados para análise.

A análise dos Domínios Ambiente e Psicológico pelas variáveis socioeconômicas, detalhada e apresentada na Tabela 2, revelou menores pontuações entre as mulheres, os adultos de 26 a 40 anos e idosos, os menos instruídos, as pessoas de menor renda e as que recebem auxílios. A diferença foi estatisticamente significativa para as variáveis renda para ambos os domínios e sexo no Domínio Ambiente ( $\mathrm{p}>\mathrm{O}, \mathrm{O} 5)$.

Tabela 2 - Escores médios do Domínio Ambiente e Domínio Psicológico do questionário WHOQOL-ıoo por variáveis socioeconômicas

\begin{tabular}{|c|c|c|c|c|c|}
\hline \multirow[b]{2}{*}{ Variável } & \multirow[b]{2}{*}{$\mathrm{n}$} & \multicolumn{2}{|c|}{ Domínio ambiente } & \multicolumn{2}{|c|}{ Domínio psicológico } \\
\hline & & Score & Valor de $p$ & Score & Valor de $p$ \\
\hline \multicolumn{6}{|l|}{ Sexo } \\
\hline Mulheres & 72 & 43,58 & & 60,76 & \\
\hline Homens & 29 & 51,11 & $0,0038^{*}$ & 66,37 & 0,0823 \\
\hline \multicolumn{6}{|l|}{ Idade } \\
\hline 18 a 25 anos & 16 & 48,65 & & 67,26 & \\
\hline 26 a 40 anos & 23 & 41,82 & & 57,77 & \\
\hline 41 a 60 anos & 41 & 46,61 & & 63,57 & \\
\hline " 60 anos & 19 & 45,85 & 0,3508 & 60,37 & 0,2043 \\
\hline \multicolumn{6}{|l|}{ Nível de Instrução } \\
\hline Menos de 9 anos & 33 & 44,91 & & 59,91 & \\
\hline 9 a 12 anos & 40 & 45,50 & & 62,88 & \\
\hline Mais de 12 anos & 28 & 47,07 & 0,7892 & 64,55 & 0,4544 \\
\hline \multicolumn{6}{|l|}{ Renda } \\
\hline D & 12 & 51,24 & & 71,25 & \\
\hline E & 82 & 44,04 & $0,0506^{*}$ & 60,53 & $0,0158^{*}$ \\
\hline \multicolumn{6}{|l|}{ Auxílio } \\
\hline $\operatorname{sim}$ & 47 & 43,65 & 0,1045 & 61,28 & \\
\hline Não & 47 & 47,70 & & 63,57 & 0,4495 \\
\hline
\end{tabular}

${ }^{*} p " 0,05$

A análise das facetas auxilia a compreender quais características influenciam o escore dos domínios (Tabela 3). Entre as oito facetas do Domínio Ambiente, as mais mal avaliadas foram Recursos Financeiros $(38,74)$ e Ambiente Físico $(41,87)$, e a mais bem avaliada foi Transporte $(54,52)$.
A Tabela 3 apresenta a avaliação das facetas do Domínio Psicológico. Entre as cinco facetas que o compõem, as mais mal avaliadas foram Sentimentos Negativos $(52,23)$ e Sentimentos Positivos $(61,39)$ e a mais bem avaliada foi Autoestima $(70,61)$. 
Tabela 3 - Médias dos Domínios e suas facetas, em ordem descendente, questionário WHOQOL-100, Conjunto Habitacional Caminho das Montanhas, São José dos Campos/SP, $2017(n=101)$

\begin{tabular}{|c|c|}
\hline Domínio I- domínio físico & 55,28 \\
\hline Dor e desconforto & 46,28 \\
\hline Energia e fadiga & 58,73 \\
\hline Sono e repouso & 60,83 \\
\hline Domínio II - domínio psicológico & 61,50 \\
\hline Sentimentos negativos & 52,23 \\
\hline Sentimentos positivos & 61,39 \\
\hline Imagem corporal e aparência & 62,37 \\
\hline Pensar, aprender, memória e concentração & 63,06 \\
\hline Autoestima & 70,61 \\
\hline Domínio III - nível de independência & 68,02 \\
\hline Atividades da vida cotidiana & 65,78 \\
\hline Dependência de medicação ou tratamentos & 66,03 \\
\hline Mobilidade & 67,31 \\
\hline Capacidade de trabalho & 72,96 \\
\hline Domínio IV - relações sociais & 61,57 \\
\hline Suporte (apoio) social & 51,61 \\
\hline Atividade sexual & 64,29 \\
\hline Relações pessoais & 68,81 \\
\hline Domínio V-meio ambiente & 45,60 \\
\hline Recursos financeiros & 38,74 \\
\hline Ambiente físico (poluição/ruído/trânsito/clima) & 41,87 \\
\hline Segurança física e proteção & 42,00 \\
\hline Ambiente no lar & 43,38 \\
\hline Cuidados de saúde e sociais: disponibilidade e qualidade & 46,35 \\
\hline Participação em, e oportunidades de recreação/lazer & 47,28 \\
\hline Oportunidades de adquirir novas informações e habilidades & 51,42 \\
\hline Transporte & 54,52 \\
\hline Domínio VI - Espiritualidade / Religião / Crenças Pessoais & 73,64 \\
\hline
\end{tabular}




\section{Discussão}

\section{População de estudo}

Nota-se baixa escolaridade, com mais de 5,0\% dos respondentes analfabetos, predominantemente idosos. Estes valores contrastam com as médias municipais do censo de 2010 (taxa de analfabetismo 2,9\% e população jovem com pelo menos nove anos de estudo, 63,2\%). Embora a população jovem acompanhe a tendência de melhora dos indicadores, os valores são inferiores à média do município. A área rural norte possui IDHM médio $(0,665)$, correspondente à segunda faixa de IDHM mais baixa dentre os seis recortes territoriais do município.

A renda é a segunda dimensão que mais contribui para o alto IDHM do município, sendo o salário médio mensal correspondente a 3,7 salários mínimos em 2016. Os dados econômicos encontrados para a população estudada quando comparados aos dados censitários revelam uma população mais pobre e menos instruída que a média municipal, decorridos sete anos da mudança habitacional.

O baixo nível de instrução e renda e a precariedade do trabalho explicam a frequente necessidade de apoio social: $50 \%$ dos moradores que responderam o questionário recebiam algum tipo de auxílio. Entre as formas de benefício citadas, a principal foi o Bolsa Família, recebido por 40 pessoas.

Os dados socioeconômicos revelam a manutenção da vulnerabilidade econômica das pessoas que participaram desse programa habitacional. As mulheres chefes de família, com baixa renda, residência em área de risco ou insalubre e nativas da cidade têm prioridade em programas de habitação social, de acordo com os critérios definidos pelas leis federal (Brasil, 2009), estadual e municipal. Observa-se que a combinação de múltiplas vulnerabilidades, como a baixa renda e instrução e o trabalho precário, se mantiveram após a mudança habitacional. Uma revisão sistemática de 40 avaliações de impacto da saúde em projetos, programas e decisões políticas relativas à habitação, realizadas nos Estados Unidos, identificou o que foi denominado "pobreza concentrada" como um determinante da saúde crítico na dimensão comunitária da habitação, e um ponto que deve receber atenção desde o desenho do projeto habitacional. Áreas periféricas, de uso residencial estrito, com população homogeneamente de baixa renda, distantes de oportunidades de trabalho e lazer associam-se a maiores índices de violência, problemas de saúde mental e doenças crônicas (NCHH, 2016).

\section{WHOQOL-100 para avaliação da qualidade de vida}

O escore de qualidade de vida $(59,84)$ é baixo em comparação aos dados publicados na produção acadêmica, que tem como público-alvo principalmente pessoas com doenças crônicas ou trabalhadores sujeitos a desgaste físico e/ou emocional. Um estudo brasileiro, realizado no Sul do país e aplicado a 241 pessoas obteve escore 68,18 de qualidade de vida (Rocha; Fleck, 2011). A amostra foi composta por 122 pacientes internados e ambulatoriais com doença crônica, provenientes de um hospital universitário, e 119 indivíduos saudáveis membros ativos de comunidades religiosas. Um estudo realizado com 607 alunos do $1^{\circ}$ ao $6^{\circ}$ ano de medicina de uma Universidade Pública Federal, no estado de São Paulo, obteve escore de 72,7 (Cunha et al., 2017). 0 valor atribuído à qualidade de vida geral foi mais elevado do que o encontrado na aplicação da forma resumida do questionário (WHOQOL-breve) a 31 imigrantes refugiados africanos no Brasil $(49,57)$ (Horta; Cruz; Carvalho, 2019). Ressalte-se que a ideia da migração se aplica aos moradores de conjuntos habitacionais. Muitas vezes, em busca de moradas acessíveis e de qualidade, as famílias passam por situação de rua e mudanças frequentes e involuntárias de endereço, constituindo um cenário de "instabilidade habitacional" ( $\mathrm{NCHH}, 2016$, p. 8) que se relaciona a pior acesso à assistência à saúde e maior prevalência de condições de saúde mental, doenças crônicas não controladas e consequências negativas da educação e saúde dos filhos em comparação a pessoas com habitação estável (NCHH, 2016).

A maior pontuação do Domínio ERCPé corroborada pela produção acadêmica. Observa-se, nos estudos brasileiros que utilizam o instrumento WHOQOL para avaliar a qualidade de vida (Borges et al., 2013; Fogaça et al., 2009; Oliveira; Morais; Sarian, 2010; 
Reis; Costa, 2010; Silva; Amadei, 2016), que esse domínio apresenta sempre os maiores valores entre os seis domínios, o que influencia a qualidade de vida, aumentando o escore geral. Relação inversa se observa no Domínio Ambiente, domínio mais mal avaliado nas diversas aplicações do instrumento na produção acadêmica (Dallalana; Batista, 2014; Fernandes et al., 2010, 2012; Rebouças et al., 2016; Reis; Costa, 2010), o que influencia a qualidade de vida, reduzindo o escore geral.

Cruz et al. (2011) utilizaram a variação breve do questionário (WHOQOL-breve) para avaliar qualidade de vida da população geral na cidade de Porto Alegre, Rio Grande do Sul. Apesar de utilizarem o instrumento reduzido e a população ter características distintas, o Ambiente foi o segundo Domínio mais mal avaliado (59,9). Ressalta-se que este foi o único artigo da revisão bibliográfica a ter como população avaliada pessoas sem patologias ou fatores ocupacionais de risco. Na avaliação de qualidade de vida dos migrantes africanos refugiados no país, o item que recebeu pior avaliação foi, igualmente, o ambiente (50,1; valor superior ao encontrado neste estudo), e a faceta mais bem avaliada foi a relativa às relações pessoais, o que indica a importância do capital social para as populações em situações de vulnerabilidade, com acesso limitado às políticas sociais (Horta; Cruz; Carvalho, 2019).

Os domínios Psicológico (61,5o) e Ambiente (45,6o) foram pontuados em valores menores aos encontrados na literatura. Rebouças et al. (2016), em pesquisa com deficientes visuais, encontraram escores de 71,69 e 48,48, respectivamente; Rocha e Fleck (2011), em pesquisa realizada com adultos com e sem problemas crônicos de saúde, encontraram escores de 62,72 e 56,91 para pacientes e 69,10 e 63,48 para indivíduos saudáveis, respectivamente. No estudo de Horta, Cruz e Carvalho (2019), os migrantes africanos pontuaram o domínio psicológico, em escala de zero a 10o, em 63,5 e o ambiente em 5o,1, valores superiores aos aferidos pelos moradores do residencial em estudo.

A forte correlação entre os domínios Psicológico e Ambiente foi também encontrada no estudo de Cruz et al. (2011), corroborando sua relação positiva. Para Kruse (2005), no campo das Ciências Sociais, o ambiente se constrói por meio da percepção do indivíduo e é permeado por valores culturais. No campo da psicologia ambiental, o ambiente é veículo de significações, simultaneamente construído e construtor do indivíduo. Este estudo reforça a relação que vem sendo analisada nesse campo, observando-se, para a população estudada, relação entre a saúde subjetiva e o ambiente em que vivem, ambos mal avaliados. Note-se que os escores atribuídos são inferiores a escores obtidos, em produções acadêmicas, para indivíduos em situação de risco biológico (com doença) ou vulnerabilidade social extrema (migrantes em cenário de exclusão social).

Os achados dos escores médios do domínio Ambiente pelas variáveis socioeconômicas são concordantes aos estudos de Cruz et al. (2011) na pior avaliação pelas variáveis sexo feminino; menor instrução e renda. A tendência à baixa avaliação do domínio Psicológico pelo sexo feminino é corroborada por Fernandes et al. (2010) e Dallalana e Batista (2014), o que configura importante indicador devido aos novos padrões familiares brasileiros em que a mulher assume o papel de chefe de família. Outro fator relevante é a pior avaliação dos itens Ambiente e Psicológico por pessoas menos instruídas, evidenciando a importância da educação para uma melhor percepção dos fatores que contribuem para a qualidade de vida. Por fim, o indicador renda, com significância estatística para ambos os domínios, expressa a importância da aplicação de medidas de apoio que assegurem o bem-estar no processo de realocação, para evitar o reforço da vulnerabilidade que caracteriza essa população.

Nas pesquisas de Fernandes et al. (2012) e Rebouças et al. (2016), a faceta Recursos Financeiros foi, igualmente, a mais mal avaliada no Domínio Ambiente, o que a configura como indicador para a percepção positiva da qualidade de vida neste domínio. Horta, Cruz e Carvalho (2019) encontraram, igualmente, a renda como faceta de maior contribuição para o baixo escore do domínio Ambiente na população africana migrante refugiada.

Estudos na área do planejamento urbano e da saúde (Ferreira, 2012; Gordia; Quadros; Campos, 2009) apontam, historicamente, que os brasileiros que vivem em condições socioeconômicas desprivilegiadas tendem a habitar regiões periféricas, ambientes de altos índices de criminalidade e precário acesso ao 
sistema educacional e à saúde, além de vivenciarem poucas oportunidades de lazer e limitado acesso à alimentação saudável ou a novas tecnologias. As variáveis identificadas por esses pesquisadores podem auxiliar a compreender a baixa pontuação observada nas facetas: Recursos Financeiros; Cuidados de Saúde e Sociais: Disponibilidade e Qualidade; Oportunidades de Adquirir Novas Informações e Habilidades; Participação em, e Oportunidades de Recreação/Lazer. Dessa forma, constata-se que o provimento de moradia não contemplou a integralidade das dimensões do habitar, impulsionando um ciclo de intensificação das vulnerabilidades já presentes nessa população e que deveriam ser reparadas.

No Domínio Psicológico, a pior avaliação da faceta Sentimentos Negativos sinaliza a resiliência dos participantes, pois percebe-se a presença de preocupações cotidianas e alternância entre sentimentos positivos e negativos. Grotberg (2013) aponta a importância da resiliência para a manutenção da saúde mental, possibilitando aos indivíduos suportarem ou mesmo serem transformados pela adversidade. É possível que a espiritualidade, domínio mais bem avaliado no questionário, contribua para a resiliência, atuando como fator externo e proporcionando disposição para enfrentar as dificuldades advindas dessas condições adversas de vida.

\section{Considerações finais}

As políticas sociais têm como propósito prover o mínimo necessário à vida digna para os cidadãos de um determinado território, reduzindo sua vulnerabilidade e aumentando sua resiliência. Políticas habitacionais, portanto, devem ser avaliadas por parâmetros mais amplos do que a qualidade estrutural da habitação.

Em estudo que analisou os programas habitacionais com financiamento internacional para a urbanização de favelas, Magalhães e Villarosa (2012) classificaram três elementos consideradas chave para o sucesso do desenho e a implantação bem sucedidos desses programas: aspectos físicos, urbanísticos e arquitetônicos do projeto; componentes sociais e de participação em sua elaboração e implantação (incluindo ações sociais intersetoriais para inclusão e/ou desenvolvimento social e o fortalecimento do capital social local); e aspectos institucionais e de gestão. A integração desses três elementos cria condições mais ou menos favoráveis para a implantação de um projeto habitacional que, de fato, responda às necessidades identificadas em uma concepção ampliada de habitação, relacionada à saúde e ao bemestar, ou seja, que considere desde as relações sociais possíveis no lar ao entorno que o circunda, inserindo o domicílio no contexto urbano e considerando o acesso ao trabalho e aos equipamentos sociais. Uma moradia adequada, para utilizar o termo diagnóstico da Secretaria de Habitação do estado de São Paulo.

A população avaliada neste estudo, embora pequena em tamanho amostral, é representativa de diversas populações-alvo de políticas e programas habitacionais no país. Guarda, no entanto, particularidades, como a falta de mobilização e organização social, elemento desfavorável para a reinvindicação de seus direitos. 0 processo de realocação também apresenta especificidades, como a falta de documento de posse definitiva passados sete anos da entrega do imóvel, fator que provavelmente influencia negativamente a subjetividade dos habitantes e se reflete na sensação de baixo apoio social e disponibilidade de cuidados sociais de qualidade.

Em comum com outras populações realocadas por políticas públicas, compartilham a situação de vulnerabilidade econômica, social e ambiental, que, a despeito de evidências de ineficácia no plano internacional, continuam a receber moradias sociais em conjuntos localizados nas periferias urbanas, distantes de equipamentos sociais e frequentemente não apoiadas por políticas sociais de reforço ao capital social, fator que comprovadamente auxiliaria a construção de identidade coletiva comunitária. Provenientes de diferentes bairros, em situação de vulnerabilidade econômica e social, é comum que as famílias, majoritariamente uniparentais, formadas por jovens mães e com alto percentual de idosos dependentes, agreguem a vulnerabilidade espacial ao seu repertório de dificuldades cotidianas.

A aplicação do instrumento WHOQOL a esta população alvo de política habitacional, que habita 
há sete anos uma área periférica de transição urbano-rural de um desenvolvido município paulista, atesta a ineficácia desta política para a percepção positiva da qualidade de vida, em especial no que se refere ao ambiente, dimensão constituinte do conceito de habitação. A baixa satisfação com as oportunidades de trabalho e renda, aspectos que compõem uma moradia saudável, se combinam ao comprometimento na subjetividade, e, ainda que demonstrando resiliência, os recursos disponíveis dos moradores ou aportados por políticas sociais não foram potentes para reduzir suas múltiplas vulnerabilidades. As dimensões avaliadas no questionário WHOQOL equiparam a percepção de qualidade de vida dos moradores àquelas percebidas por populações portadoras de doenças crônicas ou de migrantes refugiados. Ressaltadas as limitações deste estudo de caso transversal, que não considerou as patologias presentes na população, e cujos resultados não devem ser extrapolados para todas as populações alvo de políticas habitacionais. Considera-se a relevância dos achados para a avaliação objetiva da qualidade de vida, como instrumento de articulação de políticas sociais, como vem sendo realizado de modo mais sistemático em algumas iniciativas internacionais (NCHH, 2016).

Desta forma, reafirma-se a importância de um ambiente adequado de vida para o bem-estar subjetivo, individual e coletivo, e aponta-se a preocupação de este ambiente ser insatisfatório no conjunto habitacional em questão. O estudo corrobora o uso potencial do questionário WHOQOL como instrumento para a avaliação de programas que proveem habitações de interesse social, ressaltandose a importância de aplicá-lo em dois momentos, para seguimento longitudinal.

Os dados encontrados no estudo foram apresentados e discutidos com a equipe de saúde da família que assiste a área. A divulgação dos resultados à equipe de saúde da família possibilitou um olhar de fora, e a objetividade da análise favoreceu o rompimento de preconcepções e representações sociais associadas à população moradora de conjuntos habitacionais, carregada de preconceitos e julgamentos. A relação entre o ambiente, a saúde e a subjetividade dos moradores, com a mediação da universidade, puderam ser compreendidas na dimensão do cuidado.
Os dados foram igualmente apresentados à Defensoria Pública, em uma reunião com a participação de agentes públicos da área habitacional. O instrumento corroborou a demanda por qualidade de vida no conjunto habitacional para o qual as pessoas foram removidas, e com o qual ainda não se identificam socialmente.

Passados dois anos da realização do trabalho, o contato da universidade com a equipe de saúde se mantém e ações projetadas vem sendo implementadas, gradativamente, pelo poder público. A participação social permanece incipiente. A análise, associada a outros projetos de extensão universitária de integração ensino/serviço realizadas no bairro, foi um ponto de partida para ações de melhoria estrutural e de reforço do capital social do bairro, atualmente em curso, promovidas pela gestão municipal.

O instrumento WHOQOL mostrou-se adequado para diagnóstico da qualidade de vida no conjunto habitacional estudado, e sugere-se seu uso no monitoramento da política habitacional no que se refere à meta de redução da vulnerabilidade e melhoria da qualidade de vida dos cidadãos.

\section{Referências}

\section{A SECRETARIA. Secretaria de Estado da Habitação} de São Paulo, São Paulo, 2020. Disponível em: <tinyurl.com/4fn7ut7t . Acesso em: 18 abr. 2020.

BORGES, M. C. et al. Avaliação da qualidade de vida e do tratamento fisioterapêutico em pacientes com cervicalgia crônica. Fisioterapia em Movimento, Curitiba, v. 26, n. 4, p. 873-881, 2013. DOI: 10.1590/So103-51502013000400016

BRASIL. Lei $n^{0}$ 11.977, de 7 de julho de 2009. Dispõe sobre o Programa Minha Casa, Minha Vida - PMCMV e a regularização fundiária de assentamentos localizados em áreas urbanas e dá outras providências. Diário Oficial da União, Brasília, DF, 8 jul. 20o9. Disponível em: <https://bit.ly/2Q1ESxo>. Acesso em: 27 nov. 2018.

\section{CDHU - COMPANHIA DE DESENVOLVIMENTO} HABITACIONAL E URBANO. Código de conduta e integridade. São Paulo: Secretaria da Habitação do Estado de São Paulo, [2011]. 
CRUZ, L. N. et al. Quality of life in Brazil: normative values for the WHOQOL-BREF in a southern general population sample. Quality of Life Research, Berlin, v. 20, n. 7, p. 1123-1129, 2011. DOI: 10.1007/s11136-011-9845-3

CUNHA, D. H. F. et al. Percepção da qualidade de vida e fatores associados aos escores de qualidade de vida de alunos de uma escola de medicina. Jornal Brasileiro de Psiquiatria, Rio de Janeiro, v. 66, n. 4, p. 189-196, 2017. DOI: 10.159o/oo472085000000170

DALLALANA, T. M.; BATISTA, M. G. R. Qualidade de vida do cuidador durante internação da pessoa cuidada em unidade de urgência/ emergência: alguns fatores associados. Ciência e Saúde Coletiva, Rio de Janeiro, v. 19, n. 11, p. 4587-4594, 2014. DOI: 10.1590/1413812320141911.16592013

FERNANDES, J. S. et al. Qualidade de vida dos enfermeiros das equipes de saúde da família: a relação das variáveis sociodemográficas. Texto e Contexto, Florianópolis, v. 19, n. 3, p. 434-442, 2010. DOI: 10.1590/So104-07072010000300004

FERNANDES, J. S. et al. A relação dos aspectos profissionais na qualidade de vida dos enfermeiros das equipes saúde da família. Revista da Escola de Enfermagem da USP, São Paulo, v. 46, n. 2, p. 404-412, 2012. DOI: 10.159o/ Soo8o-62342012000200019

FERREIRA, J. S. W. (Coord.). Produzir casas ou construir cidades? Desafios para um novo Brasil urbano. São Paulo: Editora Fupam, 2012.

FLECK, M. P. A. et al. Aplicação da versão em português do instrumento de avaliação psicológica da Organização Mundial de Saúde (WHOQOL-10o). Revista de Saúde Pública, São Paulo, v. 33, n. 2, p. 198-205, 1999. DOI: 10.159o/ So034-89101999000200012

FOGAÇA, M. C. et al. Estresse ocupacional e suas repercussões na qualidade de vida de médicos e enfermeiros intensivistas pediátricos e neonatais. Revista Brasileira de Terapia Intensiva, São Paulo, v. 21, n. 3, p. 299-305, 2009. DOI: 10.159o/ So103-507X2009000300010
GORDIA, A. P.; QUADROS, T. M. B.; CAMPOS, W. Variáveis sociodemográficas como determinantes do domínio meio ambiente da qualidade de vida de adolescentes. Ciência e Saúde Coletiva, Rio de Janeiro, v. 14, n. 6, p. 2261-2268, 2009. DOI: $10.1590 /$ S1413-81232009000600035

GROTBERG, E. H. Nuevas tendencias en resiliencia. In: MELILLO, A.; OJEDA, E. N. S. (Ed.). Resiliencia: descubriendo las propias fortalezas. Buenos Aires: Paidós, 2013. p. 19-30, 2013. HORTA, A. L. M.; CRUZ, M. G.; CARVALHO, G. Famílias refugiadas africanas: qualidade de vida, expectativas e necessidades em relação à saúde. Saúde e Sociedade, São Paulo, v. 28, n. 4, p. 113-123, 2019. DOI: 10.1590/So104-12902019180959

KAZTMAN, R. Notas sobre la medición de la vulnerabilidad social. In: CEPAL - COMISIÓN ECONÓMICA PARA AMÉRICA LATINA Y EL CARIBE. Quinto taller regional sobre la medición de la pobreza: métodos y aplicaciones: documentos presentados. Santiago, 200o. p. 275-301.

KRUSE, L. Compreendendo o ambiente em psicologia ambiental. Psicologia USP, São Paulo, v. 16, n. 1-2, p. 41-46, 2005. DOI: 10.1590/So10365642005000100006

MAGALHÃES, F.; VILLAROSA, F. Urbanização de favelas: lições aprendidas no Brasil. Nova York: Banco Interamericano de Desenvolvimento, 2012.

NCHH - NATIONAL CENTER FOR HEALTHY HOUSING. A systematic review of Health Impact Assessments on housing decisions and guidance for future practice. Columbia, 2016.

OLIVEIRA, R. R.; MORAIS, S. S.; SARIAN, L. O. Efeitos da reconstrução mamária imediata sobre a qualidade de vida de mulheres mastectomizadas. Revista Brasileira de Ginecologia e Obstetrícia, Rio de Janeiro, v. 32, n. 12, p. 6o2-6o8, 2010. DOI: 10.1590/So100-72032010001200007

PNUD - PROGRAMA DAS NAÇÕES UNIDAS PARA O DESENVOLVIMENTO. Relatório do Desenvolvimento Humano 2014: sustentar o progresso humano, reduzir as vulnerabilidades e reforçar a resiliência. Nova York: 2014. 
REBOUÇAS, C. B. A. et al. Avaliação da qualidade de vida de deficientes visuais. Revista Brasileira de Enfermagem, Brasília, DF, v. 69, n. 1, p. 72-78, 2016. DOI: 10.1590/o034-7167.2016690110i

REIS, M. G.; COSTA, I. P. Qualidade de vida relacionada à saúde em pacientes com lúpus eritematoso sistêmico no Centro-Oeste do Brasil. Revista Brasileira de Reumatologia, v. 5o, n. 4, p. 408-422, 2010. DOI: 10.1590/So48250042010000400006

ROCHA, N. S.; FLECK, M. P. A. Avaliação de qualidade de vida e importância dada a espiritualidade/religiosidade/crenças pessoais (SRPB) em adultos com e sem problemas crônicos de saúde. Revista de Psiquiatria Clínica, São Paulo, v. 38, n. 1, p. 20-23, 2011. DOI: 10.159o/ So101-60832011000100005
SILVA, J. P.; AMADEI, J. L. Influência da atenção médica na qualidade de vida (WHOQOL-10o) de mulheres com lúpus eritematoso sistêmico. Revista Brasileira de Reumatologia, São Paulo, v. 56, n. 3, p. 198-205, 2016. DOI: 10.1016/j.rbr.2015.01.010

UNGAR, M.; BROWN, M.; LIEBENBERG, L. et al. Distinguishing differences in pathways to resilience among Canadian youth. Canadian Journal of Community Mental Health, Waterloo, v. 27, n. 1, p. 1-13, 2008.

WHO - WORLD HEALTH ORGANIZATION. WHOQOL: measuring quality of life. Geneva, 1997.

WHO - WORLD HEALTH ORGANIZATION. Developing guidance for health protection in the built environment: mitigation and adaptation. Geneva, 2010.

\section{Contribuição dos autores}

Carvalho contribuiu na concepção e desenho do estudo e na coleta, organização e análise de dados relativos à dimensão ambiental. Inocêncio contribuiu na concepção e desenho do estudo e na coleta, organização e análise de dados relativos à dimensão subjetiva. Guadagnin contribuiu na supervisão da análise e interpretação de dados do estudo, na dimensão da relação subjetividade e qualidade de vida e na revisão crítica do conteúdo intelectual do estudo. Amorim contribuiu na supervisão da análise e interpretação de dados do estudo, na dimensão da relação ambiente e qualidade de vida e na revisão crítica do conteúdo intelectual do estudo. Vianna foi responsável por todos os aspectos do estudo, assegurando as questões de precisão e integridade, bem como contribuiu na revisão crítica da versão final. Todos os autores aprovaram a versão final do estudo.

Recebido: 05/11/2020

Aprovado: $21 / 12 / 2020$ 